\title{
European Option Pricing of Fractional Black-Scholes Model Using Sumudu Transform and its Derivatives
}

\author{
Waseem Asghar Khan, Faryal Aijaz Ansari \\ Department of Mathematics and Basic Sciences, Balochistan UET Khuzdar \\ E-mail:waseemasg@gmail.com \\ Govt. Girls Higher Secondary school Khairpur \\ E-mail:faryal.aijaz@gmail.com
}

\begin{abstract}
In this work an analytical solution of Fractional Black-Scholes European option pricing equation is solved.The analytical solution is based on Sumudu Transform and its differential and integral properties. The obtained solution is presented in the form of Fractional Taylor series with easily computable components then numerical solutions are represented graphically.
\end{abstract}

Keywords: Sumudu Transform, Fractional integrals and derivatives for Sumudu Transform, Basic definitions of Fractional Calculus, Fractional Black-Scholes European option pricing equations, Fractional Sumudu Transform method..

\section{Introduction}

Fractional differential operators have a long history, having been mentioned by Leibniz in a letter to L'Hopital in 1695. However, it is in the past hundred years that the most attractive work in engineering and scientific application have been found. It is widely and efficiently used to describe many phenomena arising in engineering, physics, economy and science. A family of numerical [1-3], semi-analytical [4-5], and analytical methods has been developed for solving ordinary and fractional differential equations [6-7]. Many partial differential equations of fractional order have been studied and solved. For example many researchers studied the existence of solutions of the Black-Scholes model using many methods [8-12].

The Black-Scholes equation is one of the most significant mathematical models for a financial market. It is a second order parabolic partial differential equation that governs the value of financial derivatives. The BlackScholes model for pricing stock options has been applied to many different commodities and payoff structures. The Black-Scholes model for value of an option is described by the following equation:

$$
\frac{\partial \psi}{\partial \tau}+\frac{\sigma x^{2}}{2} \frac{\partial^{2} \psi}{\partial x^{2}}+r(\tau) x \frac{\partial \psi}{\partial x}-r(\tau) \psi=0, \quad(x, \tau) \in \mathbb{R}^{+} \times(0, T), \quad 0<q \leq 1
$$

\footnotetext{
* Corresponding Author.Waseem Asghar Khan, E-mail:waseemasg@gmail.com @ (1)
} 2010 Mathematics Subject Classification: 35R11;26A33; 35A22. 
where $\psi(x, \tau)$ is the European option price at asset price $x$ and at time $\tau, T$ is the maturity, $r(\tau)$ is the risk free interest rate and $\sigma(x, \tau)$ shows the volatility function of underlying asset. The payoff functions are:

$$
\psi_{c}(x, \tau)=\max (x-E, 0), \quad \psi_{p}(x, \tau)=\max (E-x, 0),
$$

where $\psi_{c}(x, \tau)$ and $\psi_{p}(x, \tau)$ are the price of the European call and put options, respectively. $E$ is the exercise price for the option.

In this work, we let the below given fractional Black-Scholes equation

$$
\frac{\partial^{q} \psi}{\partial \tau^{q}}+\frac{\sigma x^{2}}{2} \frac{\partial^{2} \psi}{\partial x^{2}}+r(\tau) x \frac{\partial \psi}{\partial x}-r(\tau) \psi=0 . \quad(x, \tau) \in \mathbb{R}^{+} \times(0, T) \quad 0<q \leq 1
$$

The aim of this paper is to applied the Sumudu Transform for pricing European option of Fractional version of the Black-Scholes equation by using Sumudu transform and its integral and differential properties.

\section{Sumudu Transform}

The Sumudu transform is an integral transform, first proposed by Watugala in 1998, [13] to find solution of differential equations and solve engineering problems.

The Sumudu transform is explained over the set of functions:

$$
A=\left\{\psi(\tau): \exists M, t_{1}, t_{2}>0,|\psi(\tau)|<M e^{\frac{\tau}{t_{j}}}, \quad \text { if } \tau \epsilon(-1)^{j} \times[0, \infty)\right\},
$$

by the following formula

$$
F(u)=S[\psi(\tau)]=\int_{0}^{\infty} \frac{1}{u} e^{-\tau / u} \psi(\tau) d \tau .
$$

The existence and uniqueness was given in [14]. For more information and properties of Sumudu transform and its derivatives, [14-19]. Actually one can simply prove that there is a big connection between double Sumudu and double Laplace transforms.

\section{Basic Definitions of Fractional Calculus and Sumudu Transform}

Definition 1 A real function $\psi(x), x>0$ is in the space $K_{\mu}, \mu \epsilon \mathbb{R}$ if there is a real number $\lambda>\mu$ such that $\psi(x)=x^{\lambda} g(x)$, where $g(x) \in K[0, \infty)$ and it is in the space $K_{\mu}^{m}$ if and only if $\psi^{(m)} \epsilon K_{\mu}$ for $m \in \mathbb{N}$.

Definition 2 The Riemann-Liouville fractional integral operator of order $q$ of a function $\psi(x) \epsilon K_{\mu}, \mu \geq-1$ is given as follows

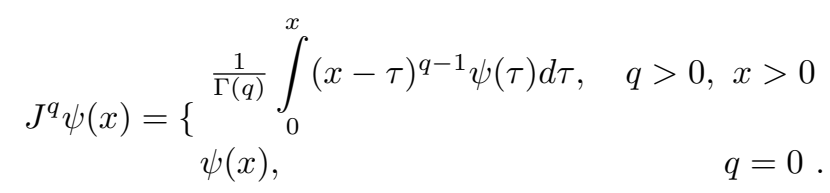

The operator $J^{q}$ has some properties, for $q, r \geq 0, \xi, \mu \geq-1$ and $C$ a real constant:

- $J^{0} \psi(x)=\psi(x)$,

- $J^{q} J^{r} \psi(x)=J^{q+r} \psi(x)$,

- $J^{q} J^{r} \psi(x)=J^{r} J^{q} \psi(x)$,

- $J^{q} x^{\xi}=\frac{\Gamma(\xi+1)}{\Gamma(q+\xi+1)} x^{q+\xi}$,

- $J^{q} C=\frac{C}{\Gamma(q+1)} x^{q}$.

Definition 3 The Caputo Fractional derivatives $D^{q}$ of a function $\psi(x)$ of any real number $q$ such that $m-1<q \leq$ $m, m \in \mathbb{N}$, for $x>0$ and $\psi \epsilon C_{-1}^{m}$ as: 


$$
D^{q} \psi(x)=\left\{\begin{array}{lr}
\frac{1}{\Gamma(m-q)} \int_{0}^{x}(x-\tau)^{m-q-1} \psi^{(m)}(\tau) d \tau \\
\frac{\partial^{m} \psi(x)}{\partial x^{m}}, & q=m,
\end{array}\right.
$$

and has the following properties for $m-1<q \leq m, m \in \mathbb{N}, \mu \geq-1$ and $\psi \epsilon C_{\mu}^{m}$

- $D^{q} J^{q} \psi(x)=\psi(x)$,

- $J^{q} D^{q} \psi(x)=\psi(x)-\sum_{k=0}^{m-1} \psi^{(k)}\left(0^{+}\right) \frac{x^{k}}{k !}$, for $x>0$.

Definition 4 The Mittag-Leffler function $E_{q}(x)$ with $q>0$ is defined by the following series:

$$
E_{q}(x)=\sum_{n=0}^{\infty} \frac{x^{n}}{\Gamma(q n+1)}
$$

Definition 5 The Sumudu transform $S[\psi(\tau)]$ of the Riemann-Liouville fractional integral is given as below [20 - 21]

$$
S\left[J^{q} \psi(\tau)\right]=u^{q} S[\psi(\tau)]
$$

Definition 6 The Sumudu transform $S[\psi(\tau)]$ of the Caputo fractional derivative is given as below [20 - 21] :

$$
S\left[D^{q} \psi(\tau)\right]=u^{-q} S[\psi(\tau)]-\sum_{k=0}^{m-1} u^{-q+k} \psi^{(k)}(0), \quad m-1<q \leq m,
$$

and the inverse sumudu transform of:

$$
S^{-1}\left[\sum_{k=0}^{m-1} u^{k} \psi^{(k)}(0)\right]=\sum_{k=0}^{m-1} \frac{\tau^{k} \psi^{(k)}(0)}{\Gamma(k+1)} .
$$

\section{Analysis of Proposed Fractional Sumudu Transform Method}

To show the fundamental scheme of this technique, we let the given below fractional PDE:

$$
D_{\tau}^{q} \psi(x, \tau)+\mathcal{L}[\psi(x, \tau)]+\aleph[\psi(x, \tau)]=Q(x, \tau), \tau>0, m-1<q \leq m,
$$

by the initial condition

$$
\psi(x, 0)=f_{0}(x),
$$

here $D_{\tau}^{q}=\partial^{q} / \partial \tau^{q}$ is the fractional Caputo derivative, $\mathcal{L}$ is the linear differential operator, $\aleph$ is the nonlinear differential operator, and $Q(x, \tau)$ is the source term of the function $\psi(x, \tau)$.

Now, both sides using the Sumudu transform (4.1),

$$
S\left[D_{\tau}^{q} \psi(x, \tau)\right]+S[\mathcal{L}[\psi(x, \tau)]+\aleph[\psi(x, \tau)]]=S[Q(x, \tau)] .
$$

Applying the differential property of Sumudu transform,

$$
S[\psi(x, \tau)]=\psi(x, 0)+u^{q} S[Q(x, \tau)-\mathcal{L}[\psi(x, \tau)]-\aleph[\psi(x, \tau)]] .
$$

Applying both sides the Sumudu inverse (4.4)

$$
\psi(x, \tau)=\psi(x, 0)+S^{-1}\left[u^{q} S[Q(x, \tau)-\mathcal{L}[\psi(x, \tau)]-\aleph[\psi(x, \tau)]]\right],
$$

Applying the integral property of Sumudu transform 


$$
\psi(x, \tau)=\psi(x, 0)+J_{\tau}^{q}[Q(x, \tau)-\mathcal{L}[\psi(x, \tau)]-\aleph[\psi(x, \tau)]] .
$$

The technique suppose a series solution for $\psi(x, \tau)$ given by

$$
\begin{aligned}
\psi_{0}(x, \tau) & =\psi(x, 0)=f_{0}(x), \\
\psi_{n+1}(x, \tau) & =J^{q}(Q(x, \tau))-J^{q}\left(\mathcal{L}\left[\psi_{n}(x, \tau)\right]\right)-J^{q}\left(\aleph\left[\psi_{n}(x, \tau)\right]\right) \\
& =\sum_{n=1}^{\infty} f_{n}(x) \frac{\tau^{n q}}{\Gamma(q n+1)},
\end{aligned}
$$

then the terms $\psi_{n}(x, \tau)$ follows:

$$
\psi(x, \tau)=\sum_{n=0}^{\infty} \psi_{n}(x, \tau)=\sum_{n=0}^{\infty} f_{n}(x) \frac{\tau^{n q}}{\Gamma(q n+1)} .
$$

This is new Fractional Sumudu Transform Method,

where the nonlinear term $\aleph[x] \psi(x, \tau)$ is decomposed by the recently developed technique depends mostly on algebraic and trigonometric identities, and also on Taylor expansions.

\section{Applications and Discussions}

In this section, we discuss the implementation of our proposed algorithm and investigate its accuracy.The simplicity and accuracy of this proposed method is illustrated through the following Fractional Black-Scholes equations.

Example 1 Let the given below fractional Black-Scholes option pricing equation [12]

$$
\frac{\partial^{q} \psi}{\partial \tau^{q}}=\frac{\partial^{2} \psi}{\partial x^{2}}+(k-1) \frac{\partial \psi}{\partial x}-k \psi, \quad 0<q \leq 1
$$

with initial condition

$$
\psi(x, 0)=\max \left(e^{x}-1,0\right) .
$$

Note that this model have just two dimensionless parameters $k=\frac{2 r}{\sigma^{2}}$, where $k$ denotes the stability between the rate of interests and the variability of stock returns and the dimensionless time to expiry $\frac{1}{2} \sigma^{2} T$, still however there are four dimensional parameters, $E, T, \sigma^{2}$ and $r$, in the original statements of the equation.

Now, applying the Fractional Sumudu transform technique with the initial condition,

$$
S[\psi(x, \tau)]=\left(\max \left(e^{x}-1,0\right)\right)+u^{q} S\left[\psi_{x x}+(k-1) \psi_{x}-k \psi\right] .
$$

Using both sides the Inverse Sumudu,

$$
\psi(x, \tau)=\max \left(e^{x}-1,0\right)+S^{-1}\left(u^{q} S\left[\psi_{x x}+(k-1) \psi_{x}-k \psi\right]\right) .
$$

Using the integral property of Sumudu transform,

$$
\psi(x, \tau)=\max \left(e^{x}-1,0\right)+J_{\tau}^{q}\left[\psi_{x x}+(k-1) \psi_{x}-k \psi\right] .
$$

The method assumes a series solution for $\psi(x, \tau)$

$$
\begin{aligned}
\psi_{0}(x, \tau) & =\psi(x, 0)=f(x), \\
\psi_{n+1}(x, \tau) & =J_{\tau}^{q}\left[\psi_{n x x}+(k-1) \psi_{n x}-k \psi_{n}\right]=\sum_{n=1}^{\infty} f_{n}(x) \frac{\tau^{n q}}{\Gamma(q n+1)},
\end{aligned}
$$

and the functions $\left(f_{k}\right)_{k=0 \ldots}$ are given by:

$$
\begin{aligned}
f_{0}= & \psi_{0}, \\
f_{1}= & f_{0 x x}+(k-1) f_{0 x}-k f_{0}, \\
f_{2}= & f_{1 x x}+(k-1) f_{1 x}-k f_{1}, \\
f_{3}= & f_{2 x x}+(k-1) f_{2 x}-k f_{2}, \\
& \vdots \\
f_{n+1}= & f_{n x x}+(k-1) f_{n x}-k f_{n} .
\end{aligned}
$$


So that the solution $\psi(x, \tau)$ in series form is defined as

$$
\psi(x, \tau)=f_{0}+f_{1} \frac{\tau^{q}}{\Gamma(q+1)}+f_{2} \frac{\tau^{2 q}}{\Gamma(2 q+1)}+f_{3} \frac{\tau^{3 q}}{\Gamma(3 q+1)}+\cdots+f_{n} \frac{\tau^{n q}}{\Gamma(n q+1)},
$$

So that the solution $\psi(x, \tau)$ of the equation is defined as

$$
\psi(x, \tau)=\sum_{n=0}^{\infty} \psi_{n}(x, \tau)=\max \left(e^{x}-1,0\right) E_{q}\left(-k \tau^{q}\right)+\max \left(e^{x}, 0\right)\left(1-E_{q}\left(-k \tau^{q}\right)\right),
$$

where $E_{q}(x)$ is Mittag-Leffler function in one parameter. For special condition $q=1$, we get

$$
\psi(x, \tau)=\max \left(e^{x}-1,0\right) e^{-k \tau}+\max \left(e^{x}, 0\right)\left(1-e^{-k \tau}\right) .
$$

which is an exact solution of the above Black-Scholes model (5.1) for $q=1$. The solution of $\psi(x, \tau)$ w.r.t $x$ and $\tau$ when $q=1$ is shown in Figure 5.1

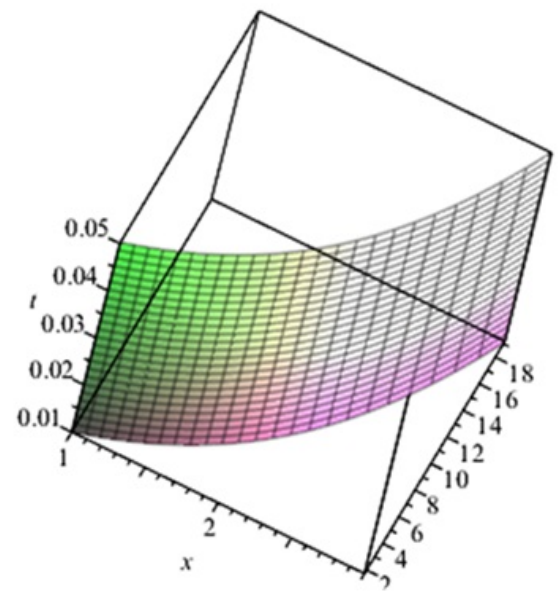

Fig 5.1: The surface represents the solution for Black-Scholes eq (5.1) w.r.t $x$ and $\tau$ for $q=1$

Example 2 In this example, let the given below generalized fractional Black-Scholes option pricing equation [10] defined as:

$$
\frac{\partial^{q} \psi}{\partial \tau^{q}}+0.08(2+\sin x)^{2} x^{2} \frac{\partial^{2} \psi}{\partial x^{2}}+0.06 x \frac{\partial \psi}{\partial x}-0.06 \psi=0, \quad 0<q \leq 1
$$

by initial condition

$$
\psi(x, 0)=\max \left(x-25 e^{-0.06}, 0\right) .
$$

Now, using the Fractional Sumudu transform method with the initial condition,

$$
S[\psi(x, \tau)]=\left(\max \left(x-25 e^{-0.06}, 0\right)\right)-u^{q} S\left[0.08(2+\sin x)^{2} x^{2} \psi_{x x}+0.06 x \psi_{x}-0.06 \psi\right] .
$$

Using both sides the Inverse Sumudu,

$$
\psi(x, \tau)=\max \left(x-25 e^{-0.06}, 0\right)-S^{-1}\left(u^{q} S\left[0.08(2+\sin x)^{2} x^{2} \psi_{x x}+0.06 x \psi_{x}-0.06 \psi\right]\right) .
$$

Operating with the integral property of Sumudu transform,

$$
\psi(x, \tau)=\max \left(x-25 e^{-0.06}, 0\right)-J_{\tau}^{q}\left[0.08(2+\sin x)^{2} x^{2} \psi_{x x}+0.06 x \psi_{x}-0.06 \psi\right] .
$$

The technique assumes a series solution for $\psi(x, \tau)$

$$
\begin{aligned}
\psi_{0}(x, \tau) & =\psi(x, 0)=f(x) \\
\psi_{n+1}(x, \tau) & =-J_{\tau}^{q}\left[0.08(2+\sin x)^{2} x^{2} \psi_{n x x}+0.06 x \psi_{n x}-0.06 \psi_{n}\right]=\sum_{n=1}^{\infty} f_{n}(x) \frac{\tau^{n q}}{\Gamma(q n+1)}
\end{aligned}
$$


and the functions $\left(f_{k}\right)_{k=0 \ldots}$ are given by:

$$
\begin{aligned}
f_{0}= & \psi_{0}, \\
f_{1}= & -\left[0.08(2+\sin x)^{2} x^{2} f_{0 x x}+0.06 x f_{0 x}-0.06 f_{0}\right], \\
f_{2}= & -\left[0.08(2+\sin x)^{2} x^{2} f_{1 x x}+0.06 x f_{1 x}-0.06 f_{1}\right], \\
f_{3}= & -\left[0.08(2+\sin x)^{2} x^{2} f_{2 x x}+0.06 x f_{2 x}-0.06 f_{2}\right], \\
& \vdots \\
f_{n+1}= & -\left[0.08(2+\sin x)^{2} x^{2} f_{n x x}+0.06 x f_{n x}-0.06 f_{n}\right] .
\end{aligned}
$$

So that the solution $\psi(x, \tau)$ in series form is defined as

$$
\psi(x, \tau)=f_{0}+f_{1} \frac{\tau^{q}}{\Gamma(q+1)}+f_{2} \frac{\tau^{2 q}}{\Gamma(2 q+1)}+f_{3} \frac{\tau^{3 q}}{\Gamma(3 q+1)}+\cdots+f_{n} \frac{\tau^{n q}}{\Gamma(n q+1)}
$$

So that the solution $\psi(x, \tau)$ of the equation is followed by

$$
\psi(x, \tau)=\sum_{n=0}^{\infty} \psi_{n}(x, \tau)=\max \left(x-25 e^{-0.06}, 0\right) E_{q}\left(-0.06 \tau^{q}\right)+x\left(1-E_{q}\left(-0.06 \tau^{q}\right)\right)
$$

where $E_{q}(x)$ is Mittag-Leffler function in one parameter. For special condition $q=1$, we get

$$
\psi(x, \tau)=\max \left(x-25 e^{-0.06}, 0\right) e^{-0.06 \tau}+x\left(1-e^{-0.06 \tau}\right) .
$$

which is an exact solution of the above Black-Scholes model (5.11) for $q=1$.

The solution of $\psi(x, \tau)$ w.r.t $x$ and $\tau$ when $q=1$ is shown in Figure 5.2.

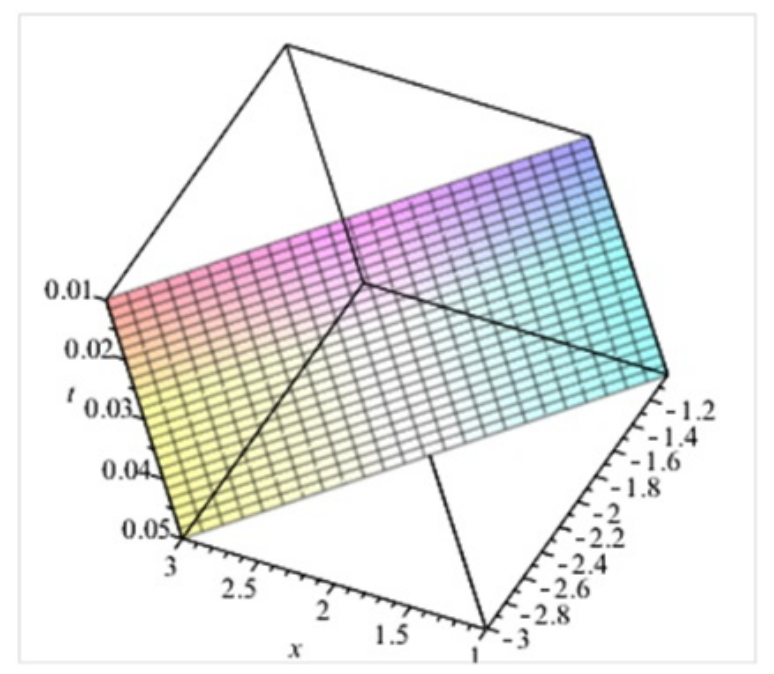

Fig 5.2: The surface represents the solution for Black-Scholes eq (5.11) w.r.t $x$ and $\tau$ for $q=1$

\section{Conclusion}

In this paper, a concept of the Sumudu transform and its derivatives is successfully applied for pricing European option of the fractional Black-Scholes equation. Two examples from the literature [22] are presented to determine the efficiency and simplicity of the proposed method.This scheme was clearly very efficient and powerful technique in finding the solutions of approximate solutions as well as numerical solutions. 


\section{References}

[1] K. Diethelm and N.J. Ford, "Multi-order fractional differential equations and their numerical solution", Applied Mathematics and Computation, Vol.154, (2004), pp.621-640.

[2] M.A.M. Ghandehari and M. Ranjbar, "A numerical method for solving a fractional partial differential equation through converting it into an NLP problem", Computers and Mathematics with Applications, Vol.65, (2013), pp.975-982.

[3] F.W. Liu, V. Anh, and I. Turner, "Numerical solution of the space fractional Fokker-Planck equation", Journal of Computational and Applied Mathematics, Vol.166, (2004), pp.209-219.

[4] J.S. Duan, R. Rach, D. Buleanu, and A.M. Wazwaz, "A review of the Adomian decomposition method and its applicaitons to fractional differential equations", Communications in Fractional Calculus, Vol.3, (2012), pp.73-99.

[5] J.H. He, "Variational iteration method a kind of non-linear analytical technique: some examples", International Journal of Non-Linear Mechanics, Vol.34, (1999), pp.699-708.

[6] X.Y. Jiang and H.T. Qi, "Thermal wave model of bioheat transfer with modified Riemann-Liouville fractional derivative", Journal of Physics, Vol.45, (2012), Article ID 485101.

[7] J.H. Ma and Y.Q. Liu, "Exact solutions for a generalized nonlinear fractional Fokker-Planck equation", Nonlinear Analysis. Real World Applications, Vol.11, (2010), pp.515-521.

[8] M. Bohner and Y. Zheng, "On analytical solutions of the Black- Scholes equation", Applied Mathematics Letters , Vol.22, 3(2009), pp.309-313.

[9] R. Company, E. Navarro, J. R. Pintos, and E. Ponsoda, "Numerical solution of linear and nonlinear Black-Scholes option pricing equations", Computers \& Mathematics with Applications, Vol.56, 3(2009), pp. 813-821.

[10] Z. Cen and A. Le, "A robust and accurate finite difference method for a generalized Black-Scholes equation", Journal of Computational and Applied Mathematics, Vol.235, 13(2011), pp. 3728-2733.

[11] J. Ankudinova and M. Ehrhardt, "On the numerical solution of nonlinear Black-Scholes equations", Computers E Mathematics with Applications, Vol.56, 3(2008), pp. 799-812.

[12] V. G“ulkac, The homotopy perturbation method for the Black- Scholes equation", Journal of Statistical Computation and Simulation, Vol.80, 12(2010), pp. 1349-1354.

[13] G. K . Watugala, "Sumudu transform - a new integral transform to solve differential equations and control engineering problems", Mathematical Engineering in Industry, Vol.6, 4(1998), pp. 319-329.

[14] A. Kılıçman and H. Eltayeb, "A note on integral transforms and partial differential equations", Applied Mathematical Sciences, Vol.4, 6(2010), pp. 109-118.

[15] M. A. Asşru, "Further properties of the Sumudu transform and its applications", International Journal of Mathematical Education in Science and Technology, Vol.33, 3(2002), pp. 441-449.

[16] F. B. M. Belgacem, A. A. Karaballi, and S. L. Kalla, " Analytical investigations of the Sumudu transform and applications to integral production equations", Mathematical Problems in Engineering , 3-4, (2003), pp. $103-118$.

[17] H. Eltayeb, A. Kılıçman, and B. Fisher, "A new integral transform and associated distributions", Integral Transforms and Special Functions, Vol.21, 5-6,(2010), pp. 367-379.

[18] A. Kılıçman, V. G. Gupta, and B. Sharma, "On the solution of fractional Maxwell equations by Sumudu transform", Journal of Mathematics Research, Vol.2, 4(2010), pp. 147-151.

[19] M. A. Asiru, "Sumudu transform and the solution of integral equations of convolution type", International Journal of Mathematical Education in Science and Technology, Vol.32, 6(2001), pp. 906-910.

[20] A . M.Wazwaz, "A new technique for calculating Adomian polynomials for nonlinear operators", Appl. Math. Comput ., 111(2000), pp. 33-51.

[21] A. M.Wazwaz, Partial Differential Equations: Methods and Applications, Balkema , Leiden, (2002).

[22] M. Madani, M. Fathizadeh, Y. Khan, and A. Yildirim, "On the coupling of the homotopy perturbation method and Laplace transformation", Mathematical and Computer Modelling, vol. 53, 9-10(2011), pp. 1937-1945. 